\title{
Klotho and Chronic Kidney Disease
}

\author{
Ming Chang Hu $u^{a, b} \cdot$ Makoto Kuro-o ${ }^{a, d} \cdot$ Orson W. Moe ${ }^{a-c}$ \\ ${ }^{a}$ Charles and Jane Pak Center for Mineral Metabolism and Clinical Research and Departments of

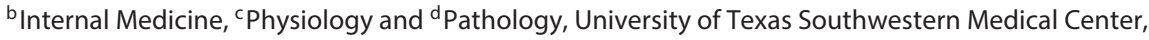 \\ Dallas, Tex., USA
}

\begin{abstract}
Through alternative splicing, Klotho protein exists both as a secreted and a membrane form whose extracellular domain could be shed from the cell surface by secretases and released into the circulation to act as endocrine factor. Unlike membrane Klotho which functions as a coreceptor for fibroblast growth factor-23 (FGF23) to modulate FGF23 signal transduction, soluble Klotho is a multifunction protein present in the biological fluids including blood, urine and cerebrospinal fluid and plays important roles in antiaging, energy metabolism, inhibition of Wnt signaling, antioxidation, modulation of ion transport, control of parathyroid hormone and $1,25(\mathrm{OH})_{2} \mathrm{VD}_{3}$ production, and antagonism of renin-angiotensin-aldosterone system. Emerging evidence from clinical and basic studies reveal that chronic kidney disease is a state of endocrine and renal Klotho deficiency, which may serve as an early biomarker and a pathogenic contributor to chronic progression and complications in chronic kidney disease including vascular calcification, cardiac hypertrophy, and secondary hyperparathyroidism. Supplementation of exogenous Klotho and/or upregulation of endogenous Klotho production by using rennin angiotensin system inhibitors, HMG $\mathrm{CoA}$ reductase inhibitors, vitamin $\mathrm{D}$ analogues, peroxisome proliferator-activated receptors-gamma agonists, or anti-oxidants may confer renoprotection from oxidation and suppression of renal fibrosis, and also on prevention or alleviation of complications in chronic kidney disease. Therefore, Klotho is a highly promising candidate on the horizon as an early biomarker, and as a novel therapeutic agent for chronic kidney disease.
\end{abstract}

Copyright $\odot 2013$ S. Karger AG, Basel

Chronic kidney disease (CKD) is a growing global public health problem and more than 26 million Americans are estimated to be affected [1,2]. There is a paucity of sensitive biomarkers to detect early CKD and there are few specific and effective strategies to retard CKD progression to end-stage renal disease and prevent or ameliorate extrarenal complications. Better biomarkers are needed for early detection and accurate prediction, and novel therapies are 
desperately needed for preserving kidney function and minimizing cardiovascular complications.

The Klotho gene was identified in 1997 when a locus was accidentally interrupted that resulted in a cluster of features resembling premature multiorgan failure $[3,4]$. Klotho is expressed the highest in the kidney and, interestingly, the phenotype of the Klotho hypomorph or knock-out mice, have striking similarities with CKD patients such as ectopic soft tissue calcification, hyperphosphatemia, and high plasma FGF23 [5], suggesting that CKD might be a state of Klotho deficiency.

Klotho is a single-pass transmembrane protein and is expressed in multiple tissues but is particularly high in the kidney $[4,6]$. In the mouse, rat, and human kidney, Klotho is strongly expressed in the distal convoluted tubules but also unequivocally found in the proximal convoluted tubule $[7,8]$ and perhaps in some inner medullary collecting cells although in lower levels compared to distal convoluted tubules [7, 9]. In addition to membrane Klotho, a secreted form of Klotho protein is generated from the Klotho gene through alternative splicing and directly released into extracellular compartment and present in multiple body fluids. A second form of soluble Klotho is ectodomain shedding of membrane Klotho from the cell surface under the effect of A Desintegrin And Metalloproteinase (ADAM) 10 and ADAM17, which is stimulated by insulin and inhibited by metalloproteinase inhibitors [10]. Soluble Klotho protein is present in cerebrospinal fluid [11], blood [5, 11], and urine of mammals $[5,8]$.

Membrane-anchored and soluble Klotho proteins likely have distinct functions. Membrane Klotho forms a complex with fibroblast growth factor (FGF) receptors and functions as a coreceptor for FGF23, a bone-derived hormone that induces negative phosphate balance through promoting renal phosphate excretion and reducing serum levels of 1,25-dihydroxyvitamin $\mathrm{D}_{3}\left(1,25-(\mathrm{OH})_{2} \mathrm{VD}_{3}\right)$ [12]. Soluble Klotho functions as an endocrine factor, and plays important roles in a variety of processes including modulation of ion transport [8, 13], Wnt signal transduction [14], anti-renin-angiotensin system [15], antisenescence [16], and antioxidation [17]. In addition, Klotho suppresses 1a-hydroxylase in the kidney to regulate calcium metabolism [18] and participates in the regulation of PTH synthesis in the parathyroid gland by FGF23 [19].

\section{Klotho Deficiency in Chronic Kidney Disease}

When one compares the features of Klotho-deficient mice with manifestations of $\mathrm{CKD}$, there are interesting similarities including hyperphosphatemia, high plasma FGF23, ectopic soft tissue calcification, and decreased Klotho in the 
Table 1. Endocrine and renal Klotho deficiency in CKD in humans and in rodent models

\begin{tabular}{|c|c|c|c|c|c|c|c|c|c|c|c|}
\hline \multirow[t]{2}{*}{$\begin{array}{l}\text { Category of } \\
\text { kidney disease }\end{array}$} & \multirow{2}{*}{$\begin{array}{l}\text { Cause or } \\
\text { animal } \\
\text { model }\end{array}$} & \multicolumn{2}{|c|}{$\begin{array}{l}\text { Renal Klotho } \\
\text { protein }\end{array}$} & \multicolumn{2}{|c|}{$\begin{array}{l}\text { Renal Klotho } \\
\text { mRNA }\end{array}$} & \multirow[t]{2}{*}{$\begin{array}{l}\text { Blood Klotho } \\
\text { assay }\end{array}$} & \multirow{2}{*}{$\begin{array}{l}\text { Urine } \\
\text { Klotho } \\
\text { assay }\end{array}$} & \multicolumn{3}{|c|}{$\begin{array}{l}\text { Human or } \\
\text { rodent }\end{array}$} & \multirow[t]{2}{*}{$\begin{array}{l}\text { Reference } \\
\text { no. }\end{array}$} \\
\hline & & IB & $\mathrm{IHC}$ & NB & qPCR & & & M & $\mathrm{R}$ & $\mathrm{H}$ & \\
\hline CKD & Npx+IRI & $\downarrow$ & $\downarrow$ & $\downarrow$ & $\downarrow$ & $\downarrow$ by IP + IB & $\downarrow$ by IB & $\sqrt{ }$ & $\sqrt{ }$ & & 5 \\
\hline CKD & $5 / 6 \mathrm{Npx}$ & & & $\downarrow$ & & & & & $\sqrt{ }$ & & 28 \\
\hline CKD & ICGN & & & & $\downarrow$ & & & & & & 24 \\
\hline CKD & Npx in apo-E-/- & $\downarrow$ & & & $\downarrow$ & $\downarrow$ by ELISA & & $\sqrt{ }$ & & & 24 \\
\hline Hypertension & SHR & & & $\downarrow$ & & & & & $\sqrt{ }$ & & 28 \\
\hline Hypertension & DOCA & & & $\downarrow$ & & & & & $\sqrt{ }$ & & 28 \\
\hline Hypertension & Ang II & $\downarrow$ & $\downarrow$ & $\downarrow$ & & & & & $\sqrt{ }$ & & 16 \\
\hline Hypertension & Ang II & $\downarrow$ & & $\downarrow$ & & & & & $\sqrt{ }$ & & 67 \\
\hline Hypertension & SHR & $\downarrow$ & $\downarrow$ & & $\downarrow$ & & & & $\sqrt{ }$ & & 27 \\
\hline$\overline{\mathrm{DM}}$ & OLETF & & & $\downarrow$ & & & & & $\sqrt{ }$ & & 28 \\
\hline $\mathrm{DM}$ & streptozotocin & $\downarrow$ & & & & & & $\sqrt{ }$ & & & 39 \\
\hline$\overline{\mathrm{DM}}$ & $d b / d b$ & $\downarrow$ & $\downarrow$ & & $\downarrow$ & & & $\sqrt{ }$ & & & 26 \\
\hline CKD & CGN & $\downarrow$ & $\downarrow$ & $\downarrow$ & & & & & & $\sqrt{ }$ & 2 \\
\hline$\overline{C K D}$ & DN & $\downarrow$ & $\downarrow$ & $\downarrow$ & & & & & & $\sqrt{ }$ & 21 \\
\hline Allograft & CGR & $\downarrow$ & $\downarrow$ & $\downarrow$ & & & & & & $\sqrt{ }$ & 21 \\
\hline CKD & $\mathrm{N} / \mathrm{A}$ & & & & & & $\downarrow$ by IB & & & $\sqrt{ }$ & 5 \\
\hline CKD & $\mathrm{N} / \mathrm{A}$ & & & & & $\uparrow$ by ELISA & & & & $\sqrt{ }$ & 35 \\
\hline Adults & $\begin{array}{l}\text { community } \\
>65 \text { years }\end{array}$ & & & & & $\begin{array}{l}\downarrow \text { by ELISA with } \\
\text { high death }\end{array}$ & & & & $\sqrt{ }$ & 33 \\
\hline Adults & $\begin{array}{l}\text { community } \\
24-102 \text { years }\end{array}$ & & & & & $\begin{array}{l}\downarrow \text { by ELISA with } \\
\text { high CVD }\end{array}$ & & & & $\sqrt{ }$ & 34 \\
\hline Children/adults & normal & & & & & $\begin{array}{l}\downarrow \text { by ELISA with } \\
\text { age }\end{array}$ & & & & $\sqrt{ }$ & 32 \\
\hline $\begin{array}{l}\text { Age/aged } \\
\text { kidney }\end{array}$ & normal & $\downarrow$ & & & & & & $\sqrt{ }$ & & & 30 \\
\hline
\end{tabular}

CGN = Chronic glomerulonephritis; CsA = cyclosporine A; CVD = cardiovascular disease; $\mathrm{DM}=$ Diabetes mellitus; $\mathrm{DN}=\mathrm{Diabetic}$ nephropathy; DOCA-salt $=$ deoxycorticosterone acetate and high salt intake (DOCA-Salt); FK = FK-506, or Tacrolimus; IB = Immunoblot; ICGN = ICR-derived spontaneous glomerulonephritis; IHC = immunohistochesimstry; IP = immunoprecipitation; $\mathrm{NB}=$ Northern blot; $\mathrm{Npx}=$ nephrectomy; OLETF = the Otsuka Long-Evans Tokushima fatty rat; RT = reverse transcription; SHR = spontaneous hypertension; qPCR = quantitative or real time PCR; CGR = chronic graft rejection.

blood and kidney, suggesting that CKD might be a state of Klotho deficiency. Experimental data and clinical findings have thus far supported this view (table 1) $[17,20-26]$.

\section{Aging Kidney}

Aging rodents (older than 2 years) have significantly higher serum creatinine $\left(\mathrm{S}_{\mathrm{Cr}}\right)$ [27] and lower renal Klotho protein expression compared to young ones [28]. Notably, the decreased renal Klotho protein levels in aged rodents is associated with increased oxidative stress, proinflammatory cytokine production, and activation of endothelin signal transduction (table 1) [27]. 
There are numerous epidemiological studies showing association of Klotho gene or promoter polymorphisms with a variety of phenotypes in several ethic populations. A few are noteworthy. Klotho gene polymorphism F352 V and/or C370 $\underline{S}$ within exon 2 increases secreted levels and $\beta$-glucosidase activity of Klotho in vitro in cultured cells, suggesting that Klotho gene variants may influence the trafficking and catalytic activity of Klotho [29]. However, one epidemiological study showed that the heterozygous genotype (Klotho-VS/WT) but not the Klotho-VS/VS is found in individuals older than 88 years [30], speaking against the VS being a simple longevity allele. In fact, one case report of a de novo gene translocation causing what was interpreted as but not yet proven to be high plasma Klotho activity, is associated with a syndrome of hypophosphatemic rickets, hyperparathyroidism, and skeletal abnormalities [31]. The pathophysiology of this presumed high Klotho state is not known.

One ELISA kit recently developed to determine plasma Klotho, found levels that are two-fold higher in normal children than adults [32]. Likewise, Semba et al. [33] used the same kit to determine plasma Klotho and found an association of low plasma Klotho with higher overall mortality in a community-based population. Furthermore, the same group found an independent association of higher plasma Klotho with low incidence of cardiovascular disease (table 1), but no relationship between plasma Klotho and renal function (eGFR) [34].

Whether and how blood Klotho is downregulated in elderly humans remains to be examined. One possibility is that loss of nephrons in the aged kidney reduces Klotho expression and shedding into circulation. Another is that renal Klotho reduction is a part of systemic Klotho reduction by an internal 'clock' which renders the kidneys more susceptible to cumulative insults.

\section{CKD Patients}

Renal Klotho RNA is markedly decreased in CKD patients with etiologies (table 1) including obstructive nephropathy, rejected transplanted kidneys, diabetic nephropathy, chronic glomerulonephritis, and unknown causes [20]. However, data on plasma Klotho levels in human CKD is limited. Using a recently generated ELISA, Yamazaki et al. showed that plasma Klotho correlated with $\mathrm{S}_{\mathrm{Cr}}, \mathrm{BUN}$ and FGF23 [32], suggesting plasma Klotho might be affected by renal function even though that study did not specifically enroll CKD patients. Using the same kit, another group showed 3- to 4-fold higher plasma Klotho in CKD patients compared to healthy volunteers (table 1) [35]. Larger multicenter cohorts with validated assays are required to define plasma Klotho levels in CKD patients.

While clinical data in human plasma Klotho is yet to be acquired, urinary Klotho levels of CKD patients were shown to decrease at a very early stage and 
sustainably reduced with progression of CKD (table 1) [5]. Klotho levels in plasma, urine and kidney are decreased in parallel in a rodent CKD model (table 1) [5] but this parallel relationship remains to be studied in humans.

\section{Rodent CKD Models}

Subtotal (5/6th) nephrectomized rats have low Klotho mRNA expression in the kidney 8 weeks after surgery (table 1) [26]. Another CKD rodent model induced by unilateral nephrectomy plus contralateral ischemic reperfusion injury ( $\mathrm{Npx}$ + IRI) followed by high dietary phosphate intake has also downregulated renal Klotho protein and mRNA expression (table 1) [5]. In addition to the moderate increase in $\mathrm{S}_{\mathrm{Cr}}$, hyperphosphatemia, and anemia, ectopic calcification is observed in soft tissues including the kidney, aorta, and heart. Apolipoprotein Edeficient $\left(a p o-E^{-/}\right)$mice rendered uremic by uninephrectomy and contralateral electrocautery have lower renal Klotho expression compared to apo- $E^{-/-}$nonuremic mice (table 1) [22]. The degree of reduction in Klotho is similar in magnitude in plasma, kidney, and urine [5] indicating that CKD is a stalte of 'panKlotho' deficiency. One implication is that measurement of urinary Klotho might be a simpler and easier surrogate to plasma Klotho.

In addition to CKD due to ablation of renal mass, rodents with immunemediated chronic glomerulonephritis also have reduced renal Klotho mRNA (table 1) [17]. Klotho overexpression improves renal function and ameliorates renal histology, which are associated with less superoxide anion generation and lipid peroxidation, and with decreased levels of cell senescence markers, mitochondrial DNA fragmentation, and apoptosis. Thus, Klotho protein might serve as a renoprotective factor by diminishing oxidative stress, cell senescence and apoptosis [17].

The OLETF rat, a model for the metabolic syndrome, has lower Klotho mRNA in the kidneys (table 1) $[23,26]$. The administration of the peroxisome proliferator-activated receptor- $\gamma$ agonist, thiazolidinedione, increases renal Klotho mRNA, attenuates abnormal lipid and glucose metabolism, and reduces systolic blood pressure [23]. Whether thiazolidinedione directly stimulates renal Klotho expression on this model is not known but it is conceivable that the increase in Klotho is what preserves kidney function and improves lipid metabolism.

Klotho protein in the kidney is decreased in a streptozotocin-induced diabetic nephropathy model (table 1) [36]. Both insulin and phloridzin correct hyperglycemia, reverse renal Klotho reduction, and improve kidney function and histology. An in vitro study showed that high glucose medium incubation suppresses Klotho expression in canine kidney cells. Insulin is able to shed extracellular domain of Klotho [10] and may increase Klotho release into the blood which may be a novel mechanism of insulin action in alleviation of diabetic ne- 
phropathy. In another diabetic mouse with insulin resistance ( $d b / d b)$, renal expression of Klotho mRNA and protein is also significantly decreased (table 1) [24]. An in vitro study revealed that methylglyoxal-modified albumin reduces Klotho mRNA and protein in cultured renal tubular cells [24]. Klotho supplementation or overexpression in cultured cells inhibits NF- $\mathrm{KB}$ activation and TNF- $\alpha$-induced production of inflammatory cytokines such as IL-8, IL-6, regulated upon activation normal $\mathrm{T}$ cell expressed and presumably secreted (RANTES), and monocyte chemoattractant protein-1 [24].

In spontaneous hypertensive rats and in rats treated with deoxycorticosterone and high-salt diet, renal Klotho mRNA expression is dramatically reduced (table 1) [26]. Klotho gene delivery attenuates the hypertension and ameliorates renal damage in spontaneously hypertensive rats [25] which is associated with decreased superoxide production in kidneys and aortas [25].

Renal angiotensin II (Ang II) plays a pathogenic role in the downregulation of renal Klotho expression [15]. Overexpression of Klotho via gene transfer attenuates Ang II-induced functional and structural kidney damage [15]. Interestingly, this improvement is not associated with blocking Ang II-induced hypertension. In vitro study confirmed that Ang II blockade reverses Ang II-induced downregulation of Klotho expression in kidney cell line [37], providing direct evidence to support an association of renoprotection from Ang II-induced damage with Klotho upregulation [38].

\section{Mechanisms of Klotho Deficiency}

It is likely that loss of renal mass alone would completely explain the decreased production of anynephrogenicsubstance such as erythropoietin, $1,25-(\mathrm{OH})_{2} \mathrm{VD}_{3}$, and Klotho. The pathophysiological mechanisms underlying Klotho downregulation in CKD are more complex than just renal tissue loss. It is likely that uremic toxins and abnormal mineral metabolism contribute to downregulation of renal Klotho and subsequent decrease in blood Klotho (fig. 1a).

\section{Aberrant Mineral Metabolism}

Hyperphosphatemia is a pathogenic intermediate contributing to chronic progression and complications in CKD [39]. Phosphate overload can inhibit Klotho expression in the kidney (fig. 1a). Normal mice fed a high Pi diet have dramatically decreased Klotho protein and mRNA in the kidney [unpubl. data], while Klotho hypomorphic mice fed with a low Pi diet regain part of their Klotho expression [40]. Whether phosphate modulates Klotho ectodomain shedding from membrane Klotho is unknown. 


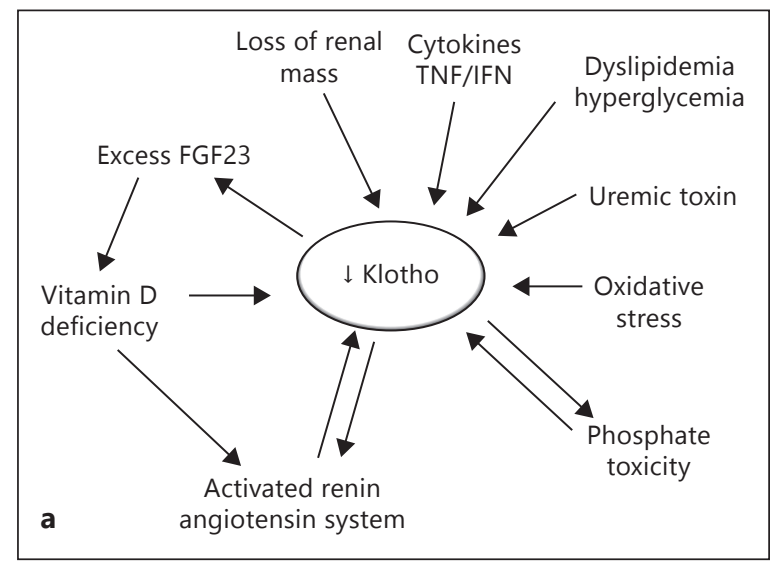

Fig. 1. Mechanisms and pathophysiological origin of Klotho deficiency in CKD. a Putative mechanism of downregulation of Klotho expression in CKD: in CKD/end-stage renal disease, multiple potential factors including loss of renal mass, overproduction of proinflammatory cytokines such as tumor necrosis factor (TNF) and interferon (IFN), dyslipidemia and hyperglycemia, elevation of uremic toxin such as indoxyl sulfate, and oxidative stress, all downregulate renal Klotho and consequently blood Klotho. There are several positive loops. Klotho deficiency stimulates FGF23 synthesis which is also enhanced by elevation of blood Pi. High blood FGF23 suppresses $1,25-(\mathrm{OH})_{2} \mathrm{VD}_{3}$ synthesis and worsens VD deficiency, which further inhibits Klotho. Low blood 1,25- $(\mathrm{OH})_{2} \mathrm{VD}_{3}$ not only reduces Klotho expression, but also stimulates renin angiotensin system which further suppresses Klotho production. Downregulation of Klotho in the kidney and the circulation further activates renin angiotensin system and decreases renal Klotho expression. On the other hand, low Klotho expression aggravates phosphate retention by decreasing urinary Pi excretion. High Pi per se also decreases renal Klotho expression.

Low 1,25-dihydroxyl-vitamin D3 $\left(1,25-(\mathrm{OH})_{2} \mathrm{VD}_{3}\right)$ may be a consequence of loss of renal tissue and high blood FGF23 in CKD (fig. 1a) [41]. Vitamin Dresponsive elements are present in human and rodent Klotho gene and administration of $1,25-(\mathrm{OH})_{2} \mathrm{VD}_{3}$ induces renal Klotho expression in vivo [18]. In vitro studies indicate that $1,25-(\mathrm{OH})_{2} \mathrm{VD}_{3}$ induces mRNA of both membrane and secreted forms of Klotho mRNA in kidney cell lines [7]. Consistent with this point, vitamin D receptor (VDR) null mice have decreased renal Klotho mRNA expression, suggesting that an interplaying network of vitamin D, phosphate and FGF23 contributes to Klotho production (fig. 1a).

\section{Uremic Toxin}

Indoxyl sulfate, a uremic toxin which accumulates in the blood in $\mathrm{CKD}$, likely promotes progression and cardiovascular disease in CKD [42, 43], and suppresses renal Klotho mRNA expression in Dahl salt-resistant normotensive and hypertensive rats [44] and induces tubulointerstitial fibrosis and increases cell senescence 


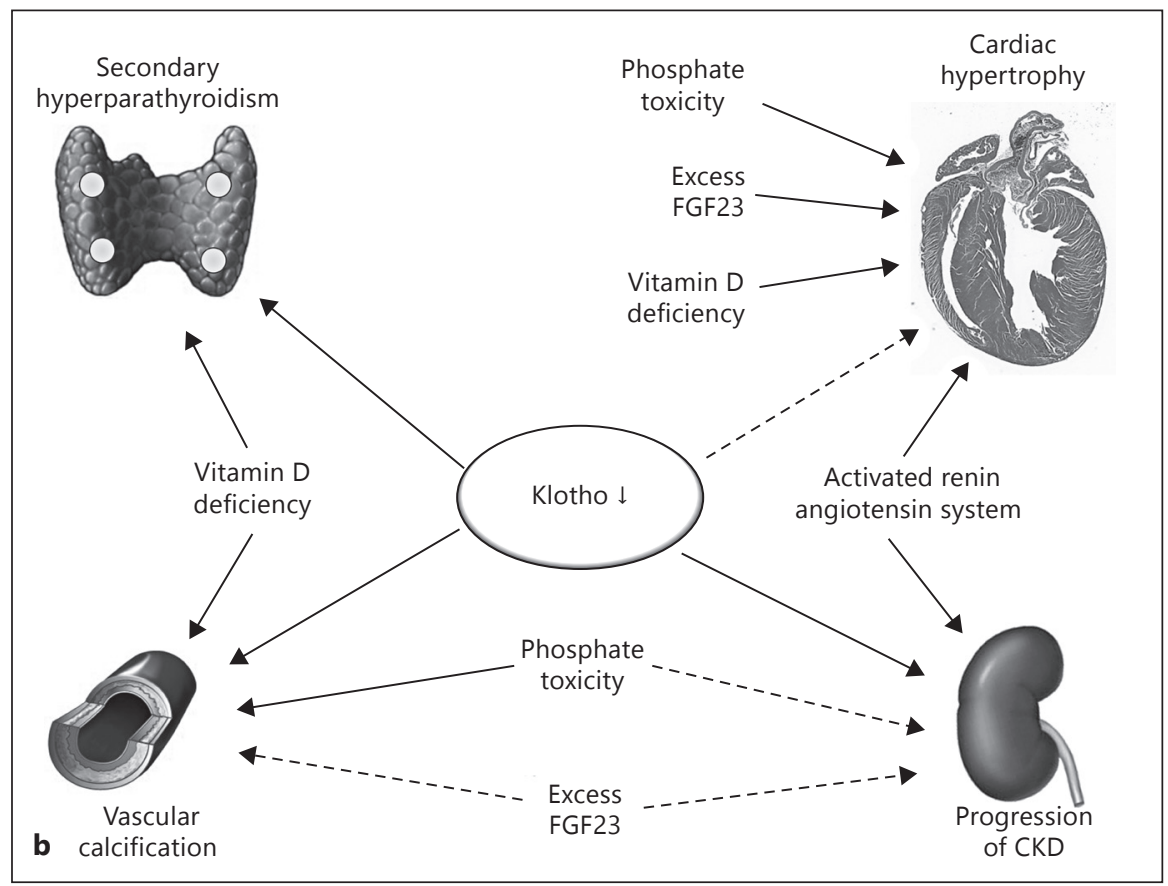

Fig. 1. b Pathogenic role of Klotho deficiency and interaction with other mineral metabolism disturbances in chronic kidney diseases: in the uremic parathyroid gland, FGFR1 and Klotho expression are downregulated which renders parathyroid gland resistant to the suppressive effect of FGF23 which triggers and promotes secondary hyperparathyroidism. The high blood phosphate and FGF23, activated renin-angiotensin system activation, and low blood vitamin D in concert contribute to cardiomyopathy. Whether Klotho plays a direct role in cardiac hypertrophy remains to be clarified (dashed line). High phosphate induces vascular smooth muscle cells reprogramming to osteoblast/chondrocytes, low blood Klotho contributes to failure of suppression of high phosphate-induced calcification. The direct effect of FGF23 on vascular calcification remains to be explored (dashed line). Low blood vitamin D accelerates vascular calcification. Low blood and renal Klotho facilitate renal fibrogenesis, impair tissue regeneration, and consequently promote chronic progression of CKD. Whether and how high FGF23 and phosphate in blood induces renal fibrosis is still unknown (dashed line).

in renal tubular cells of Dahl salt-resistant hypertensive rats [45]. Antioxidant and NF- $\kappa \mathrm{B}$ inhibitors reverse the suppressive effect of indoxyl sulfate on Klotho expression in rats and HK2 cells. Indoxyl sulfate may downregulate renal Klotho in CKD (fig. 1a) through stimulation of oxidative pathways and NF- $\kappa B$ [44].

\section{Renin-Angiotensin-Aldosterone (RAA) System}

Ang II and aldosterone suppress Klotho expression in the kidney and kidney cell lines [46]. Long-term infusion of Ang II increases blood pressure and down- 
regulates renal Klotho mRNA and protein. Administration of norepinephrine [15] or inhibitor of NO synthesis [6] cause a comparable hypertensive effect, but do not affect renal Klotho expression. Low-dose Ang II does not affect systolic blood pressure, but reduces renal Klotho mRNA in rats [15]. In addition, both the angiotensin type I receptor antagonist losartan, and the vasodilator hydralazine block the Ang II-induced rise in blood pressure, but only losartan blocks the Ang II-induced decrease in Klotho. In vivo Klotho gene transfer protects the kidney against AII-induced damage (fig. 1a) [15]. Losartan also upregulates the expression of Klotho and improves renal histological alteration in mice of chronic cyclosporine nephropathy [47].

It was proposed that Ang II-induced oxidative stress contributes to downregulation of Klotho expression, because free radical scavenging prevents Klotho depletion by both oxidative stress [9] and Ang II infusion [15]. The interplay between RAA activation, phosphate, and the vitamin D-FGF23 axis may amplify the downregulation of Klotho expression in CKD (fig. 1a). VDR null mice have hypertension and cardiac hypertrophy [48], higher renin expression and plasma Ang II compared to WT mice. In WT mice, inhibition of $1,25-(\mathrm{OH})_{2} \mathrm{VD}_{3}$ synthesis leads to increase in renin expression, whereas $1,25-(\mathrm{OH})_{2} \mathrm{VD}_{3}$ injection suppresses renin [49]. Hence, $1,25-(\mathrm{OH})_{2} \mathrm{VD}_{3}$ is a negative endocrine regulator of the RAA system (fig. 1a). Active vitamin $\mathrm{D}_{3}$ upregulates Klotho expression in mouse kidney [18] and in HEK 293 cells [46], and reverses aldosteroneinduced Klotho mRNA reduction [46]. Therefore, combination of RAA inhibition and supplementation of active vitamin $\mathrm{D}_{3}$ may provide synergistic effects on upregulation of Klotho expression.

\section{Role of Klotho Deficiency in Progression of Chronic Kidney Disease}

Klotho deficiency appears not to be a mere biomarker for CKD but pathogenic for CKD development, progression and complications (fig. 1b). To elaborate on this notion, we will examine the possible ways by which Klotho deficiency can affect the progression of CKD.

\section{Rending Renal Tissues More Vulnerable to Injury}

$K_{\text {lotho }}{ }^{--}$mice have increased senescence of progenitor cells [14]. Knock-down of endogenous Klotho promotes senescence in primary human fibroblasts [16] and exogenous Klotho decreases senescence in endothelial cells [50] and fibroblasts [16]. Cell senescence induced by Klotho depletion may be mediated by upregulation of Wnt signal activity [14]. Administration of exogenous Wnt could accelerate cell senescence in vivo and in vitro. Soluble Klotho binds to various Wnt family mem- 
bers and inhibits their biological activity [14]. One recent study shows that intracellular Klotho suppresses cell senescence by inhibiting retinoic acid-inducible geneI-induced expression of IL-6 and IL-8 both in vitro and in vivo [51].

Cell senescence is a complicated process present not only in normal aging but also in pathophysiological states $[17,52]$. Klotho deficiency in CKD would enhance cell senescence induced by oxidative stress $[17,52]$ and accelerated senescence and resultant apoptosis and stem cell depletion might decrease the kidney's ability to defend against renal insults and impair regeneration.

\section{Promote Renal Fibrogenesis}

Renal fibrosis is a prominent histological characteristic in CKD, and likely contributes to chronic progression; and TGF- $\beta_{1}$ is a major player in renal fibrosis [53]. The renal fibrosis in unilateral ureteral obstruction (UUO) is accompanied by upregulation of TGF- $\beta_{1}$, and downregulation of Klotho mRNA and protein. These alterations are exaggerated in $\mathrm{Klotho}^{+/-}$mice [54]. Klotho alleviates renal fibrosis induced by UUO and suppresses expression of fibrosis markers and TGF- $\beta_{1}$ target genes (Snail, Twist), by inhibiting TGF- $\beta_{1}$ binding to type-II TGF- $\beta$ receptor [54]. Furthermore, Klotho suppresses TGF- $\beta_{1}$-induced Smad2 phosphorylation in a rat tubular epithelial cell line suggesting that Klotho protein inhibits fibrosis primarily through inhibiting TGF- $\beta_{1}$ signaling [54].

The combination of promotion of renal fibrogenesis and retardation of renal tissue regeneration can potentially promote chronic progression (fig. lb) and Klotho supplementation can impart beneficial impact in preventing and slowing down CKD progression.

\section{Contributions of Klotho Deficiency to Complications of Chronic Kidney Disease}

An emerging hypothesis is that Klotho deficiency not only worsens renal disease but also exacerbates extra-renal complications in CKD.

\section{Ectopic Calcification}

Cardiovascular calcification is a major underlying factor for high mortality and morbidity in CKD [55]. Vascular calcification is a dynamic process resulting from the imbalance between promoters and inhibitors, FGF23 and Klotho are novel contributors to ectopic calcification in soft tissues including aorta (fig. 1b) $[4,5,56,57]$.

$K l o t h o^{-/-}$mice have extensive ectopic calcification in soft tissue which is also observed in CKD subjects, suggesting a potential pathogenic association between 
Klotho deficiency and calcification. Augmentation of Klotho by supplementation or genetic manipulation significantly inhibits vascular calcification in CKD animals [5]. Elevated plasma Pi correlates with vascular calcification in CKD, and multiple pathogenetic roles of high Pi have been proposed to induce or exacerbate vascular calcification: (1) high ambient $\mathrm{Pi}$ induces vascular smooth muscle cells reprogramming to a phenotype resembling osteoblast and osteochondrocyte in a type $3 \mathrm{Na}^{+}$-coupled transporters (Pit1 and Pit2)-dependent manner [58], and (2) high Pi causes cell apoptosis and death and resultant vesicle release for deposition on the matrix [59]. Klotho was shown to suppress this reprogramming induced by a high Pi in rat cell lines in vitro [5]. Klotho also serves as an anti-inflammatory modulator [24] in vascular endothelial cells and smooth muscle cells [16, 52].

FGF23-deficient mice have ectopic calcification in soft tissues including vasculature, raising the interesting notion that FGF23 may be protective for the vasculature. However, the high blood FGF23 in CKD patients challenges this model. One possible explanation might be that the target organs lose their response to FGF23 in CKD. Klotho and FGFR1/3 expression are downregulated when human aorta derived-smooth muscle cells are incubated with high phosphate and calcium. This concept was reinforced by the recent in vitro finding of expression of the FGF23, FGFR1/3 and Klotho in human aorta [56, 57]. Vascular smooth muscle cells undergoing osteogenic/chondrogenic trans-differentiation under the effect of uremic milieu including high Pi lose the ability to respond to FGF23 (fig. 1b) [56]. Another possibility is that extremely high FGF23 may exert pathological action via activation of off-target genes to induce vascular calcification in the setting of low blood Klotho.

\section{Uremic Cardiomyopathy}

Cardiac hypertrophy, especially left ventricular hypertrophy, is frequently encountered in CKD patients. In addition to traditional risk factors, novel factors including RAA system activation [60], vitamin D deficiency [61], high FGF23 [62], and low Klotho [62] are implicated in the cardiomyopathy of CKD (fig. 1b).

In the heart, Klotho is expressed solely at the sinoatrial node [3]. The high rate of sudden death of $\mathrm{Klotho}^{-/-}$mice under stress may partly be caused by dysrhythmia from sinoatrial node dysfunction [3]. However, no degenerative structural change was found in sinoatrial node of $\mathrm{Klotho}^{-/-}$mice, suggesting that Klotho may help maintain the functional integrity of the sinoatrial node [3]. Whether and how soluble Klotho functions as regulator of the heart pacemaker is still unclear.

The association of Klotho deficiency and cardiac hypertrophy was recently shown [62]. This study documented that even heterozygous Klotho haploinsuuficiency is associated with left ventricular hypertrophy which is not hypertension-dependent. Elevated cardiac or/and systemic FGF23 is a direct intermediate, 
because both intramyocardial and intravenous injection of FGF23 result in cardiac hypertrophy in WT mice, and in an established animal model of CKD, treatment with an FGF-receptor blocker attenuates cardiac hypertrophy, but does not change blood pressure [62]. These results unveil a Klotho-independent role for FGF23 in the pathogenesis of cardiac hypertrophy. In addition to FGF23, aberrant vitamin D and phosphate metabolism and cardiac RAA activation in Klotho deficiency situation may also participate in cardiac hypertrophy in CKD (fig. 1b).

\section{Secondary Hyperparathyroidism}

In addition to aberrant mineral metabolism (Pi and Ca, vitamin D), high FGF23 and low Klotho may contribute to secondary hyperparathyroidism. CKD patients have elevated plasma level and activity of FGF23 [62] and low FGFR1 and Klotho expression in parathyroid gland [63]. However, one study did find increased Klotho protein in uremic parathyroid gland, which may reflect Klotho expression at the very early stage of secondary hyperparathyroidism [64].

In the physiological setting with normal Klotho and FGFR expression, FGF23 decreases PTH production, increases expression of both the parathyroid calciumsensing receptor and the vitamin $\mathrm{D}$ receptor, and suppresses cell proliferation [65]. In contrast, in the uremic setting with low Klotho and FGFR1 expression in parathyroid glands, FGF23 loses its inhibitory actions on the gland, and fails to increase calcium-sensing receptor and vitamin D receptor [65]. Moreover, FGF23 administration fails to reduce blood PTH levels in CKD animals [66], indicating parathyroid resistance to FGF23, which may result from low expression of FGFR1 and Klotho in uremic hyperplastic parathyroid glands (fig. 1b) [65].

\section{Conclusion and Perspectives}

With the recent escalation of research of Klotho on renal physiology and pathophysiology, the understanding of the relationship of Klotho with kidney disease has been advanced considerably. Animal data and clinical observation to date is overwhelmingly strong to indicate that Klotho is not merely an early biomarker for CKD, but also a pathogenetic intermediate for CKD progression and complications development. Animal studies unequivocally showed that Klotho supplementation may provide novel therapy for acute kidney injury patients to retard or block its progression to CKD and for CKD by slowing progression as well as preventing and reversing extra-renal complications.

The database on plasma Klotho levels in human CKD calls for robust, validated, standardized, and high-throughput assay(s) suitable for large-scale population-based cross-sectional and longitudinal studies. The publications thus 


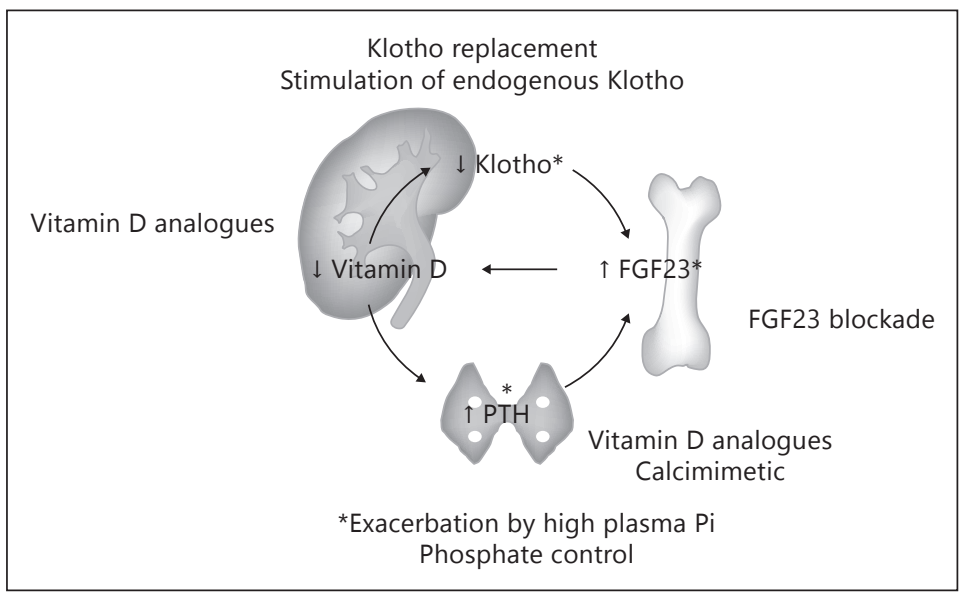

Fig. 2. Proposed therapeutic strategies in CKD. Five principal mineral disturbances in CKD: Klotho deficiency, excess FGF23, secondary hyperparathyroidism, vitamin D deficiency, and phosphate retention are shown as interacting and self-amplifying setting off a vortex of downhill spiral. Shown in italics are means to interrupt these pathways at specific points. Each maneuver has the potential of breaking the spiral. In a given patient, a particular combination of prescriptions can be tailored to best suit that individual. The prescription will also not be static in the sense that it has to be constantly readjusted with time as the patient's pathophysiology evolves and responds to therapy.

far, while informative, are from cross-sectional study and from single center with small sample size and to date use assays that cannot yet be standardized. Plasma or urinary Klotho should be explored as markers for presence of renal disease, predictors for progression, and extra-renal complications, particularly soft tissue calcification.

Beside the important observational database, it is critical to know how to translate the preclinical therapeutic results of Klotho in animal experiments to human CKD. Identification of indications for Klotho treatment will be challenging. When should we start Klotho treatment in CKD patients? As low renal Klotho and urinary Klotho may be present in very earlier stage of CKD, it is reasonable to start Klotho administration as early as possible. If that is the case, what are the therapeutic targets and how does one monitor therapy, since the effects of Klotho is so pleiotropic. Can Klotho replacement be still beneficial in advanced CKD or ERSD? Perhaps the extrarenal effects of Klotho still warrant its replacement even if there are no renal effects or salvage.

The most important concept is that we will likely individualize therapy in mineral disturbances in CKD. Figure 2 depicts a model where the principal mineral disturbances in CKD amplify each other thus creating a self-intensifying vortex that spirals downhill accelerating renal demise. In a given patient at 
a particular stage, one can envision that interruption of one or more factors may take precedence over others. Klotho supplementation therapy will most likely be part of a more complex regimen that is tailored to a particular patient.

It is likely that Klotho downregulation in CKD/ERSD is multifactorial (fig. 1) and each patient has different etiology of kidney disease and complication, and may have specific factor(s) that downregulate Klotho expression. Thus in addition to direct supplementation of Klotho protein, control or removal of those negative factors may synergistically increase Klotho efficacy or decrease Klotho dosage. Thus individualized regimens may include Klotho, renin-angiotensin system inhibitors, HMG CoA reductase inhibitors, vitamin D analogues, peroxisome proliferatoractivated receptor- $\gamma$ agonists, or antioxidants to enhance endogenous Klotho.

\section{References}

1 Coresh J, Selvin E, Stevens LA, Manzi J, Kusek JW, Eggers P, Van Lente F, Levey AS: Prevalence of chronic kidney disease in the United States. JAMA 2007;298:2038-2047.

$\checkmark 2$ Levey AS, Atkins R, Coresh J, Cohen EP, Collins AJ, Eckardt KU, Nahas ME, Jaber BL, Jadoul M, Levin A, Powe NR, Rossert J, Wheeler DC, Lameire N, Eknoyan G: Chronic kidney disease as a global public health problem: approaches and initiatives: a position statement from Kidney Disease Improving Global Outcomes. Kidney Int 2007;72:247-259.

3 Takeshita K, Fujimori T, Kurotaki Y, Honjo H, Tsujikawa H, Yasui K, Lee JK, Kamiya K, Kitaichi K, Yamamoto K, Ito M, Kondo T, Iino S, Inden Y, Hirai M, Murohara T, Kodama I, Nabeshima Y: Sinoatrial node dysfunction and early unexpected death of mice with a defect of klotho gene expression. Circulation 2004;109:1776-1782.

4 Kuro-o M, Matsumura Y, Aizawa H, Kawaguchi H, Suga T, Utsugi T, Ohyama Y, Kurabayashi M, Kaname T, Kume E, Iwasaki H, Iida A, Shiraki-Iida T, Nishikawa S, Nagai R, Nabeshima YI: Mutation of the mouse klotho gene leads to a syndrome resembling ageing. Nature 1997;390:45-51.

$5 \mathrm{Hu}$ MC, Shi M, Zhang J, Quinones H, Griffith C, Kuro-o M, Moe OW: Klotho deficiency causes vascular calcification in chronic kidney disease. J Am Soc Nephrol 2011;22:124-136.

6 Ohyama Y, Kurabayashi M, Masuda H, Nakamura T, Aihara Y, Kaname T, Suga T, Arai M, Aizawa H, Matsumura Y, Kuro-o M, Nabeshima Y, Nagail R: Molecular cloning of rat klotho cDNA: markedly decreased expression of klotho by acute inflammatory stress. Biochem Biophys Res Commun 1998;251:920-925.

$\checkmark 7$ Forster RE, Jurutka PW, Hsieh JC, Haussler CA, Lowmiller CL, Kaneko I, Haussler MR, Kerr Whitfield G: Vitamin D receptor controls expression of the anti-aging klotho gene in mouse and human renal cells. Biochem Biophys Res Commun 2011;414:557-562.

$>8 \mathrm{Hu}$ MC, Shi M, Zhang J, Pastor J, Nakatani T, Lanske B, Razzaque MS, Rosenblatt KP, Baum MG, Kuro-o M, Moe OW: Klotho: a novel phosphaturic substance acting as an autocrine enzyme in the renal proximal tubule. FASEB J 2010;24:3438-3450.

$\checkmark 9$ Mitobe M, Yoshida T, Sugiura H, Shirota S, Tsuchiya K, Nihei H: Oxidative stress decreases klotho expression in a mouse kidney cell line. Nephron Exp Nephrol 2005;101:e67-e74.

10 Chen CD, Podvin S, Gillespie E, Leeman SE, Abraham CR: Insulin stimulates the cleavage and release of the extracellular domain of Klotho by ADAM10 and ADAM17. Proc Natl Acad Sci USA 2007;104:19796-19801.

$\checkmark 11$ Imura A, Iwano A, Tohyama O, Tsuji Y, Nozaki K, Hashimoto N, Fujimori T, Nabeshima Y: Secreted Klotho protein in sera and CSF: implication for post-translational cleavage in release of Klotho protein from cell membrane. FEBS Lett 2004;565:143-147. 
-12 Urakawa I, Yamazaki Y, Shimada T, Iijima K, Hasegawa H, Okawa K, Fujita T, Fukumoto S, Yamashita T: Klotho converts canonical FGF receptor into a specific receptor for FGF23. Nature 2006;444:770-774.

13 Chang Q, Hoefs S, van der Kemp AW, Topala $\mathrm{CN}$, Bindels RJ, Hoenderop JG: The beta-glucuronidase klotho hydrolyzes and activates the TRPV5 channel. Science 2005;310:490-493.

14 Liu H, Fergusson MM, Castilho RM, Liu J, Cao L, Chen J, Malide D, Rovira II, Schimel D, Kuo CJ, Gutkind JS, Hwang PM, Finkel T: Augmented Wnt signaling in a mammalian model of accelerated aging. Science 2007;317: 803-806.

15 Mitani H, Ishizaka N, Aizawa T, Ohno M, Usui S, Suzuki T, Amaki T, Mori I, Nakamura Y, Sato M, Nangaku M, Hirata Y, Nagai R In vivo klotho gene transfer ameliorates angiotensin II-induced renal damage. Hypertension 2002;39:838-843.

16 de Oliveira RM: Klotho RNAi induces premature senescence of human cells via a p53/ p21 dependent pathway. FEBS Lett 2006;580: 5753-5758.

17 Haruna Y, Kashihara N, Satoh M, Tomita N, Namikoshi T, Sasaki T, Fujimori T, Xie P, Kanwar YS: Amelioration of progressive renal injury by genetic manipulation of Klotho gene. Proc Natl Acad Sci USA 2007;104:2331-2336.

$>18$ Tsujikawa H, Kurotaki Y, Fujimori T, Fukuda K, Nabeshima Y: Klotho, a gene related to a syndrome resembling human premature aging, functions in a negative regulatory circuit of vitamin D endocrine system. Mol Endocrinol 2003;17:2393-2403.

19 Silver J, Naveh-Many T: FGF23 and the parathyroid glands. Pediatr Nephrol 2010;25: 2241-2245.

20 Koh N, Fujimori T, Nishiguchi S, Tamori A, Shiomi S, Nakatani T, Sugimura K, Kishimoto T, Kinoshita S, Kuroki T, Nabeshima Y: Severely reduced production of klotho in human chronic renal failure kidney. Biochem Biophys Res Commun 2001;280:1015-1020.

21 Hu MC, Kuro-o M, Moe OW: Klotho and kidney disease. J Nephrol 2010;23(suppl 16): S136-S144

22 Yu J, Deng M, Zhao J, Huang L: Decreased expression of klotho gene in uremic atherosclerosis in apolipoprotein E-deficient mice. Biochem Biophys Res Commun 2010;391: 261-266.
23 Yamagishi T, Saito Y, Nakamura T, Takeda S, Kanai H, Sumino H, Kuro-o M, Nabeshima Y, Kurabayashi M, Nagai R: Troglitazone improves endothelial function and augments renal klotho mRNA expression in Otsuka Long-Evans Tokushima Fatty (OLETF) rats with multiple atherogenic risk factors. Hypertens Res 2001;24:705-709.

24 Zhao Y, Banerjee S, Dey N, LeJeune WS, Sarkar PS, Brobey R, Rosenblatt KP, Tilton RG, Choudhary S: Klotho depletion contributes to increased inflammation in kidney of the $d b / d b$ mouse model of diabetes via RelA (serine)536 phosphorylation. Diabetes 2011; 60:1907-1916

25 Wang Y, Sun Z: Klotho gene delivery prevents the progression of spontaneous hypertension and renal damage. Hypertension 2009;54:810-817.

26 Aizawa H, Saito Y, Nakamura T, Inoue M, Imanari T, Ohyama Y, Matsumura Y, Masuda H, Oba S, Mise N, Kimura K, Hasegawa A Kurabayashi M, Kuro-o M, Nabeshima Y, Nagai R: Downregulation of the Klotho gene in the kidney under sustained circulatory stress in rats. Biochem Biophys Res Commun 1998;249:865-871.

27 Zuo Z, Lei H, Wang X, Wang Y, Sonntag W, Sun Z: Aging-related kidney damage is associated with a decrease in klotho expression and an increase in superoxide production. Age (Dordr) 2011;33:261-274.

28 Manya H, Akasaka-Manya K, Endo T: Klotho protein deficiency and aging. Geriatr Gerontol Int 2010;10(suppl 1):S80-S87.

29 Arking DE, Krebsova A, Macek M Sr, Macek M Jr, Arking A, Mian IS, Fried L, Hamosh A, Dey S, McIntosh I, Dietz HC: Association of human aging with a functional variant of klotho. Proc Natl Acad Sci USA 2002;99:856-861.

30 Invidia L, Salvioli S, Altilia S, Pierini M, Panourgia MP, Monti D, De Rango F, Passarino G, Franceschi C: The frequency of Klotho KLVS polymorphism in a large Italian population, from young subjects to centenarians, suggests the presence of specific time windows for its effect. Biogerontology 2010;11:67-73.

-31 Brownstein CA, Adler F, Nelson-Williams C, Iijima J, Li P, Imura A, Nabeshima Y, ReyesMugica M, Carpenter TO, Lifton RP: A translocation causing increased alpha-klotho level results in hypophosphatemic rickets and hyperparathyroidism. Proc Natl Acad Sci USA 2008;105:3455-3460. 
32 Yamazaki Y, Imura A, Urakawa I, Shimada T, Murakami J, Aono Y, Hasegawa H, Yamashita T, Nakatani K, Saito Y, Okamoto N, Kurumatani N, Namba N, Kitaoka T, Ozono K, Sakai T, Hataya H, Ichikawa S, Imel EA, Econs MJ, Nabeshima Y: Establishment of sandwich ELISA for soluble alpha-Klotho measurement: Age-dependent change of soluble alphaKlotho levels in healthy subjects. Biochem Biophys Res Commun 2010;398:513-518.

33 Semba RD, Cappola AR, Sun K, Bandinelli S, Dalal M, Crasto C, Guralnik JM, Ferrucci L: Plasma klotho and mortality risk in older community-dwelling adults. J Gerontol A Biol Sci Med Sci 2011;66:794-800.

-34 Semba RD, Cappola AR, Sun K, Bandinelli S, Dalal M, Crasto C, Guralnik JM, Ferrucci L: Plasma klotho and cardiovascular disease in adults. J Am Geriatr Soc 2011;59:1596-1601.

-35 Sugiura H, Tsuchiya K, Nitta K: Circulating levels of soluble alpha-Klotho in patients with chronic kidney disease. Clin Exp Nephrol 2011;15:795-796.

36 Cheng MF, Chen LJ, Cheng JT: Decrease of Klotho in the kidney of streptozotocin-induced diabetic rats. J Biomed Biotechnol 2010;2010:513853.

-37 Zhou Q, Lin S, Tang R, Veeraragoo P, Peng $\mathrm{W}, \mathrm{Wu}$ R: Role of fosinopril and valsartan on klotho gene expression induced by angiotensin II in rat renal tubular epithelial cells. Kidney Blood Press Res 2010;33:186-192.

38 de Cavanagh EM, Inserra F, Ferder L: Angiotensin II blockade: a strategy to slow ageing by protecting mitochondria? Cardiovasc Res 2011;89:31-40.

39 Kendrick J, Chonchol M: The role of phosphorus in the development and progression of vascular calcification. Am J Kidney Dis 2011;58:826-834.

40 Morishita K, Shirai A, Kubota M, Katakura Y, Nabeshima Y, Takeshige K, Kamiya T: The progression of aging in klotho mutant mice can be modified by dietary phosphorus and zinc. J Nutr 2001;131:3182-3188.

41 Liu S, Tang W, Zhou J, Stubbs JR, Luo Q, Pi M, Quarles LD: Fibroblast growth factor 23 is a counter-regulatory phosphaturic hormone for vitamin D. J Am Soc Nephrol 2006;17: 1305-1315.

42 Lekawanvijit S, Adrahtas A, Kelly DJ, Kompa AR, Wang BH, Krum H: Does indoxyl sulfate, a uraemic toxin, have direct effects on cardiac fibroblasts and myocytes? Eur Heart J 2010;31:1771-1779.
43 Barreto FC, Barreto DV, Liabeuf S, Meert N, Glorieux G, Temmar M, Choukroun G, Vanholder R, Massy ZA: Serum indoxyl sulfate is associated with vascular disease and mortality in chronic kidney disease patients. Clin J Am Soc Nephrol 2009;4:1551-1558.

44 Shimizu H, Bolati D, Adijiang A, Adelibieke Y, Muteliefu G, Enomoto A, Higashiyama Y, Higuchi Y, Nishijima F, Niwa T: Indoxyl sulfate downregulates renal expression of Klotho through production of ROS and activation of nuclear factor-kB. Am J Nephrol 2011;33:319-324.

-45 Adijiang A, Shimizu H, Higuchi Y, Nishijima F, Niwa T: Indoxyl sulfate reduces klotho expression and promotes senescence in the kidneys of hypertensive rats. J Ren Nutr 2011;21:105-109.

46 Tang C, Pathare G, Michael D, Fajol A, Eichenmuller M, Lang F: Downregulation of Klotho expression by dehydration. Am J Physiol Renal Physiol 2011;301:F745-F750.

-47 Yoon HE, Ghee JY, Piao S, Song JH, Han DH, Kim S, Ohashi N, Kobori H, Kuro-o M, Yang CW: Angiotensin II blockade upregulates the expression of Klotho, the anti-ageing gene, in an experimental model of chronic cyclosporine nephropathy. Nephrol Dial Transplant 2011;26:800-813.

48 Xiang W, Kong J, Chen S, Cao LP, Qiao G, Zheng W, Liu W, Li X, Gardner DG, Li YC: Cardiac hypertrophy in vitamin $\mathrm{D}$ receptor knockout mice: role of the systemic and cardiac renin-angiotensin systems. Am J Physiol Endocrinol Metab 2005;288:E125-E132.

49 Li YC, Kong J, Wei M, Chen ZF, Liu SQ, Cao LP: 1,25 -Dihydroxyvitamin $\mathrm{D}(3)$ is a negative endocrine regulator of the renin-angiotensin system. J Clin Invest 2002;110:229-238.

50 Maekawa Y, Ohishi M, Ikushima M, Yamamoto K, Yasuda O, Oguro R, Yamamoto-Hanasaki H, Tatara Y, Takeya Y, Rakugi H: Klotho protein diminishes endothelial apoptosis and senescence via a mitogen-activated kinase pathway. Geriatr Gerontol Int 2011;11:510-516.

51 Liu F, Wu S, Ren H, Gu J: Klotho suppresses RIG-I-mediated senescence-associated inflammation. Nat Cell Biol 2011;13:254-262.

52 Nakano-Kurimoto R, Ikeda K, Uraoka M, Nakagawa Y, Yutaka K, Koide M, Takahashi T, Matoba S, Yamada H, Okigaki M, Matsubara $\mathrm{H}$ : Replicative senescence of vascular smooth muscle cells enhances the calcification through initiating the osteoblastic transition. Am J Physiol Heart Circ Physiol 2009; 297:H1673-H1684. 
53 Zeisberg M, Hanai J, Sugimoto H, Mammoto T, Charytan D, Strutz F, Kalluri R: BMP-7 counteracts TGF-betal-induced epithelial-tomesenchymal transition and reverses chronic renal injury. Nat Med 2003;9:964-968.

54 Doi S, Zou Y, Togao O, Pastor JV, John GB, Wang L, Shiizaki K, Gotschall R, Schiavi S, Yorioka N, Takahashi M, Boothman DA, Kuro-o M: Klotho inhibits transforming growth factor-beta1 (TGF-beta1) signaling and suppresses renal fibrosis and cancer metastasis in mice. J Biol Chem 2011;286:8655-8665.

55 Kendrick J, Chonchol MB: Nontraditional risk factors for cardiovascular disease in patients with chronic kidney disease. Nat Clin Pract Nephrol 2008;4:672-681.

56 Lim K, Lu TS, Molostvov G, Lee C, Lam FT, Zehnder D, Hsiao LL: Vascular klotho deficiency potentiates the development of human artery calcification and mediates resistance to fibroblast growth factor 23. Circulation 2012;125:2243-2255.

57 Donate-Correa J, Mora-Fernandez C, Martinez-Sanz R, Muros-de-Fuentes M, Perez H, Meneses-Perez B, Cazana-Perez V, NavarroGonzalez JF: Expression of FGF23/KLOTHO system in human vascular tissue. Int J Cardiol 2011, E-pub ahead of print.

58 Lau WL, Festing MH, Giachelli CM: Phosphate and vascular calcification: Emerging role of the sodium-dependent phosphate cotransporter PiT-1. Thromb Haemost 2010; 104:464-470.

-59 Shroff RC, McNair R, Skepper JN, Figg N, Schurgers LJ, Deanfield J, Rees L, Shanahan CM: Chronic mineral dysregulation promotes vascular smooth muscle cell adaptation and extracellular matrix calcification. J Am Soc Nephrol 2010;21:103-112.

60 Edwards NC, Steeds RP, Stewart PM, Ferro CJ, Townend JN: Effect of spironolactone on left ventricular mass and aortic stiffness in early-stage chronic kidney disease: a randomized controlled trial. J Am Coll Cardiol 2009; 54:505-512.
61 Achinger SG, Ayus JC: The role of vitamin D in left ventricular hypertrophy and cardiac function. Kidney Int Suppl 2005;S37-S42.

62 Faul C, Amaral AP, Oskouei B, Hu MC, Sloan A, Isakova T, Gutierrez OM, AguillonPrada R, Lincoln J, Hare JM, Mundel P, Morales A, Scialla J, Fischer M, Soliman EZ, Chen J, Go AS, Rosas SE, Nessel L, Townsend RR, Feldman HI, St John Sutton M, Ojo A, Gadegbeku C, Di Marco GS, Reuter S, Kentrup D, Tiemann K, Brand M, Hill JA, Moe OW, Kuro OM, Kusek JW, Keane MG, Wolf M: FGF23 induces left ventricular hypertrophy. J Clin Invest 2011;121:4393-4408.

63 Komaba H, Goto S, Fujii H, Hamada Y, Kobayashi A, Shibuya K, Tominaga Y, Otsuki N, Nibu K, Nakagawa K, Tsugawa N, Okano T, Kitazawa R, Fukagawa M, Kita T: Depressed expression of Klotho and FGF receptor 1 in hyperplastic parathyroid glands from uremic patients. Kidney Int 2010;77:232-238.

64 Hofman-Bang J, Martuseviciene G, Santini MA, Olgaard K, Lewin E: Increased parathyroid expression of klotho in uremic rats. Kidney Int 2010.

65 Canalejo R, Canalejo A, Martinez-Moreno JM, Rodriguez-Ortiz ME, Estepa JC, Mendoza FJ, Munoz-Castaneda JR, Shalhoub V, Almaden Y, Rodriguez M: FGF23 fails to inhibit uremic parathyroid glands. J Am Soc Nephrol 2010;21:1125-1135.

66 Lafage-Proust MH: Does the downregulation of the FGF23 signaling pathway in hyperplastic parathyroid glands contribute to refractory secondary hyperparathyroidism in CKD patients? Kidney Int 2010;77:390-392.

-67 Saito K, Ishizaka N, Mitani H, Ohno M, Nagai R: Iron chelation and a free radical scavenger suppress angiotensin II-induced downregulation of klotho, an anti-aging gene, in rat. FEBS Lett 2003;551:58-62.

Orson W. Moe, MD

Charles and Jane Pak Center of Mineral Metabolism and Clinical Research

Department of Internal Medicine, University of Texas Southwestern Medical Center at Dallas

5323 Harry Hines Blvd, Dallas, TX 75390-8885 (USA)

E-Mail orson.moe@utsouthwestern.edu 